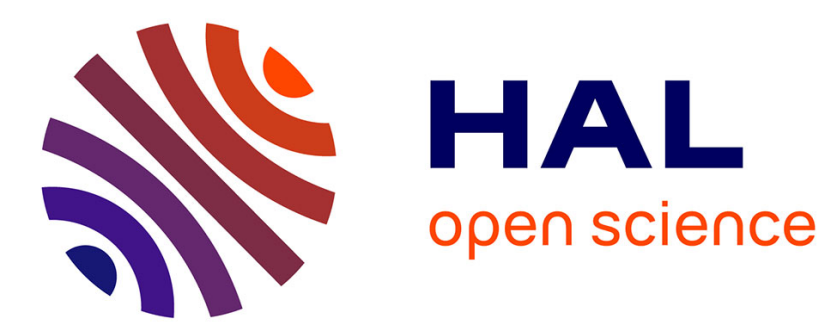

\title{
Réflexivité et culture de l'écrit. Eléments pour une conception réflexive de la littératie
}

\author{
Muriel Molinié
}

\section{To cite this version:}

Muriel Molinié. Réflexivité et culture de l'écrit. Eléments pour une conception réflexive de la littératie. Cahiers de sociolinguistique, 2009, Réflexivité, herméneutique. Vers un paradigme de recherche?, 14, pp.103-128. hal-01476549

HAL Id: hal-01476549

https://hal-univ-paris3.archives-ouvertes.fr/hal-01476549

Submitted on 24 Feb 2017

HAL is a multi-disciplinary open access archive for the deposit and dissemination of scientific research documents, whether they are published or not. The documents may come from teaching and research institutions in France or abroad, or from public or private research centers.
L'archive ouverte pluridisciplinaire HAL, est destinée au dépôt et à la diffusion de documents scientifiques de niveau recherche, publiés ou non, émanant des établissements d'enseignement et de recherche français ou étrangers, des laboratoires publics ou privés. 
Article publié dans :

Réflexivité, herméneutique. Vers un paradigme de recherche?

Sous la direction de Didier de Robillard

Cahiers de sociolinguistique $\mathrm{n}^{\circ}$ 14, Presses Universitaires de Rennes, 2009 (ps : 103-128)

\title{
Réflexivité et culture de l'écrit \\ Eléments pour une conception réflexive de la littératie
}

\author{
Muriel Molinié, Université de Cergy-Pontoise \\ EA 1392, CRTF, LaSCod
}

\begin{abstract}
La seule variété me paie, et la possession de la diversité
Montaigne

« De la vanité » (III, 9, 1540).
\end{abstract}

Lors du colloque que nous avions intitulé en 2005 avec Marie-France Bishop et Jacques David, Autobiographie et réflexivité, (Molinié, Bishop, 2006), plusieurs contributions montraient le rôle de l'écriture dans l'élaboration d'un paradigme réflexif permettant aux sujets (en formation et en recherche), de penser leurs pluralités identitaires en maillant cellesci avec des savoirs (langagiers, anthropologiques, etc.), non seulement ouverts à cette pluralité mais suffisamment puissants pour la théoriser sans la réduire.

Cette réflexion reprise trois ans plus tard, confirme qu'à nos yeux, une recherche sur les cultures de l'écrit contribue à l'élaboration d'un paradigme réflexif en éducation et en formation, dans la perspective sociodidactique des langues et des cultures qui est la notre.

Cette confirmation s'accompagne d'une prise de conscience. Nous sommes frappée de constater l'articulation pourtant étroite dans nos travaux de recherche entre littératie et réflexivité. Comme l'indique Didier de Robillard, «si construire l'autre constitue une part de l'activité de recherche, cela signifie donc que la construction de soi en fait partie » $(2007,23)$. Il est donc temps pour nous, d'interroger cette construction en déconstruisant «l'évidence » jusqu'ici non interrogée, de ce lien. Comment s'est-il noué dans notre propre histoire ellemême reliée à - et emboitée dans - une histoire sociale, des mouvements littéraires et des contextes culturels?

Explorer cette question est un processus long, qui passe par un certain nombre d' étapes. La première consistera à reprendre ici l'histoire du développement d'une culture de la personnalité que nous avions déjà amorcée dans un texte antérieur (Molinié, 1993). Nous consacrerons un premier développement (1ère partie) à Montaigne parce qu'il ouvre avec Les Essais des voies qui permettent de situer la réflexivité (telle que nous la concevons), dans une historicité. En effet, Montaigne nous indique tout d'abord, qu'un texte réflexif, peut être (durablement !) reçu comme un texte d'idées venant enrichir nos connaissances sur l'homme et la société. Il montre ensuite que la prise en compte du divers et des altérités est centrale dans cette élaboration et dans cette démarche textuelle. Il nous rappelle enfin que l'invention de formes et de genres textuels est nécessaire pour traduire et représenter cette diversité car chez lui, la «volubilité souple et inquiète» de son esprit se fait l'écho de l'irréductible diversité de la réalité.

Mais une partie seulement de notre propos est là. Car en (se) «regardant »(dans) Montaigne, on mesure à quel point la dimension éthique et politique est une condition nécessaire à l'activité réflexive. En effet, l'émancipation vis-à-vis du dogme religieux, la recherche de savoir nouveau et l'exploration de la subjectivité, sont des thématiques fondatrices de l'œuvre de Montaigne. Son exploration d'un monde «intérieur» qui 
s'individualise vis-à-vis du holisme religieux (Kauffmann, 2001) est indissociable du dispositif réflexif que constituent les Essais. Ceux-ci constituent une médiation sémiotique dans le sens où ici, le langage loin d'être une simple traduction de la pensée, est partie prenante de l'élaboration intellectuelle elle-même, inachevée, mobile, en mouvement.

Ce qui nous conduit au cœur du « sujet» (au double sens qu'a ce terme : le sujet de cet article et le sujet qui écrit ce texte).

En effet, s'il est une conviction qui anime nos pratiques (dans le champ de la formation-action et dans celui de la recherche-action), c'est bien la suivante : le langage peut, dans certaines conditions, être autre chose que la seule traduction d'une pensée. Aujourd'hui, cette conviction n'est partagée ni dans toutes les spécialités (en sciences humaines), ni dans l'ensemble des disciplines scolaires (certaines d'entre elles sont en effet fondées sur une conception transitive du langage, envisagé comme simple notation de la pensée et déterminé par cette dernière $\left.{ }^{1}\right)$. Nous observons pour notre part, que lorsqu'ils ont hérité d'une telle culture éducative, l'entrée dans une production textuelle réflexive ${ }^{2}$ est ressentie comme une nouvelle forme d'acculturation (ou encore, un niveau supérieur de littératie ${ }^{3}$ ) par des étudiants et par des praticiens-chercheurs, peu habitués à faire converser dans un même écrit savoirs théoriques et savoirs d'action, savoirs expérientiels et savoirs existentiels.

Ceci est d'autant plus paradoxal qu'à l'inverse, l'affirmation d'un continuum entre « culture de la personnalité » et «culture savante » est une thématique centrale, dans l'histoire que nous allons retracer ci-dessous. Revendiquant cette histoire, nous situons pour notre part, la problématique de «la» vérité scientifique à l'opposé tant du subjectivisme (et de ses dérives spiritualistes), que d'une scientificité établissant une frontière aussi artificielle que dogmatique entre le sujet (humain) du savoir et l'objet (humain) de ce savoir. C'est la raison pour laquelle nous attribuons aux écrits réflexifs un rôle clé dans les formations (initiale et continue) que nous conduisons dans le domaine de la professionnalisation aux métiers de la transmission et de la relation (formation d'enseignants, de directeurs de structures de formation, de travailleurs sociaux, de cadres de l'éducation et de l'accompagnement).

1 Cette conception du langage est renforcée par les approches behavioristes en éducation, qui traitent l'apprentissage comme un type particulier de conditionnement fondé sur le modèle expérimental (stimulus/réponse/renforcement) et dont on pourrait mesurer et contrôler les effets en mesurant la quantité d'objectifs atteints; en évaluant (c'est-à-dire en contrôlant) les processus d'apprentissage à travers des productions écrites standardisées (Lainé, 1998, 74).

2 Que celle-ci prenne forme à l'intérieur de démarches de type Portfolio ou dans tout autre texte de récit, d'analyse et de théorisation de pratiques socio-éducatives à visée formative et professionnalisante.

3 La littératie a été définie de façon «fonctionnelle» comme la capacité à comprendre et utiliser l'information écrite dans les activités quotidiennes (maison, travail, collectivités) pour atteindre ses objectifs, parfaire ses connaissances et accroître son potentiel (Enquêtes internationales sur la littératie des adultes - EILA-, OCDE, 2000). Pour certains chercheurs de ce domaine, ce terme réfère non seulement à la culture de l'écrit mais aussi à l'usage et au maniement potentiel du langage, qui se développe suite à une familiarisation avec la langue décontextualisée propre à l'écrit, qui a un impact considérable sur la croissance cognitive de l'enfant. L'apprentissage de l'écrit modifie le traitement du langage chez l'individu ainsi que son fonctionnement cognitif en général. (Hamers, 1997). Les recherches menées en France, dans ce champ tentent de comprendre la complexité des pratiques d'écrit dans leur diversité et leurs interrelations ; comment s'articulent les différents usages, privés et sociaux, quotidiens et exceptionnels, intellectuels et artistiques de l'écrit; et comment s'articulent les différentes compétences langagières de l'écrit, de la lecture et de l'écriture (Barré de Miniac, Brissaud, Rispail, 2004, Préface) 
Parvenue à ce point de notre étude, nous serons mieux en mesure de définir les liens entre réflexivité, diversité et cultures de l'écrit. Il s'agirait en lisant, en écrivant, d'élaborer un rapport (à soi, à autrui, à l'existence, à l'expérience, aux savoirs) qui permet à la fois de se relier (au sens de s'acculturer, se socialiser, s'historiciser, se resituer), de s'individualiser (au sens de s'autonomiser, de s'émanciper, de se séparer), d'objectiver des pratiques (sociales, professionnelles, apprenantes et, plus largement, existentielles) tout en subjectivant les savoirs vus, lus et entendus.

Concernant ce dernier point, il nous paraît fondamental de constater que, là encore, nous sommes héritiers d'une longue tradition que Michel Foucault notamment, a pris le soin d'analyser et dont nous pointerons les éléments clés dans les première et deuxième parties de cet article. Passage incontournable pour démontrer la profondeur des relations entre réflexivité et culture de l'écrit et qu'au-delà du passeur qu'est devenu, pour nous, Montaigne, elles continuent à former une part intime de notre humanité post-moderne... à condition que nous sachions les renouveler tout en nous transformant.

\section{Ecriture, éthique et culture de la personnalité dans le monde gréco-romain.}

Dans les Actes de la journée de recherche déjà mentionnée en début d'introduction, nous avons situé la réflexivité comme désignant le lien dynamique entre langage et pensée, entre pratique langagière située et activité cognitive, selon une perspective qui ne réduit pas l'activité langagière à la simple transcription (ou traduction) passive de la pensée, voire à un transfert mécanique d'information. Cette position s'expérimente empiriquement dans des domaines où les enjeux d'élaboration identitaire de l'apprenant (dans le champ éducatif) ou de l'auteur (dans le champ littéraire) sont considérés comme étant consubstantiels à l'acte d'écrire. Cette position se situe dans une tradition intellectuelle qui revendique l'idée selon laquelle celui qui écrit, c'est celui qui ressent, qui vit et affirme que le sentiment de vivre, loin d'être immédiatement donné, se développe par un travail qui ne consiste pas seulement à convertir en mots le vécu mais à «faire parler ce qui est senti» (Rouxel, 2006). Un paradigme réflexif implique donc la conjonction entre expérimentation formelle (que celle-ci se construise par l'écriture ou par toute autre médiation sémiotique), et expérience de soi. C'est le récit de cette conjonction que nous souhaitons faire ici, afin de situer les ramifications humanistes de la réflexivité et de proposer aux lecteurs de cet article de situer leurs propres dynamiques réflexives dans une histoire culturelle et un mouvement d'idées.

\subsection{L'acculturation à l'écrit : indispensable à l'entrainement de soi}

L'hellénisme donne naissance à partir $\mathrm{du}^{\mathrm{IV}} \mathrm{V}^{\circ}$ siècle avant J.C. à une conscience nationale des Grecs qui embrasse le monde européen et asiatique. En même temps que la conscience s'ouvre à un vaste horizon d'Etats, de peuples et de religions, s'éveille l'intérêt pour une culture de la personnalité qui allie le sentiment de la valeur personnelle au sens de l'individualité des autres. L'être singulier voit ses particularités empiriques prendre de l'importance et les biographies ou autobiographies le représentent jusque dans les détails les plus insignifiants de son intimité. Mais il prend plus d'importance encore comme être capable, par sa propre raison, de s'intégrer à la raison universelle pour trouver ainsi son bonheur dans une affirmation de soi ne dépendant plus d'aucune autorité. " C'est dans cette conception que se ramasse le suprême effort de la philosophie hellénistique » dont il sortit une éthique (Friedrich, op.cit., 69). La sagesse devient accessible à tous car elle n'est pas liée au savoir et à la spéculation 
théologiques ou philosophiques. Quelles que soient les fins poursuivies par les différentes doctrines de la sagesse, (stoïcisme, épicurisme et pyrrhonisme), elles ont toutes en commun d'aspirer à l'indépendance de la personne vis-à-vis du monde extérieur. Plus important encore pour l'avenir, cette philosophie éthique, (qui parle des passions et élabore des maximes pour les diriger ou les éliminer), pénètre le domaine de la vie intérieure. Ce qui vient s'ajouter ensuite, (éthique stoïcienne ou épicurienne), à ce dévoilement de l'espace intérieur est « un appendice qui pèse moins dans la balance que le dévoilement lui-même » (ibid., 70). Grâce à différents auteurs (Horace, Virgile, Tacite, Sénèque, Epictète et Lucrèce), cette culture de la sagesse se dote d'un support d'expression et de communication qui épouse la méditation libre, sans cesse recommencée, et aura les qualités requises pour pénétrer efficacement dans la pratique quotidienne de la vie d'un public élargi. "La lettre, la diatribe, le dialogue, les recueils de maximes et d'apophtegmes cultivent une forme ouverte et concise aux figures travaillées avec art, qui passent les unes dans les autres sans frontières nettement marquées » (ibid., 71). Leurs traits, (horizon cosmopolite, esprit tourné vers la culture de la personnalité, prééminence de la sagesse préoccupée de bonheur individuel, conciliation éclectique des différentes écoles, forme ouverte de la pensée et du style), passeront à la postérité. Nous les retrouverons interprétés et transformés chez Montaigne, excepté l'idéalisme éthique qui fait place chez lui à une considération descriptive de la personnalité.

A l'époque où Sénèque écrivait ses Lettres à Lucilius, le stoïcisme avait déjà depuis le $\mathrm{II}^{\circ}$ siècle av. J.C., pénétré dans l'aire de la civilisation romaine et s'était dépouillé de la dureté ascétique de ses débuts. Le philosophe pouvait estimer la sagesse possible aussi au sein d'une société cultivée, dans la vie de tous les jours dirigée par la raison. En dirigeant la conscience d'autrui, en prêtant son attention et sa sollicitude aux dimensions naturelles de la vie, Sénèque se tournait vers ceux qui s'efforcent, se sont mis en route et progressent.

Pour cela, deux procédés leur étaient proposés : l'écriture de lettres et la prise de notes ou hypomnemata. C'est sur cette dernière méthodologie que nous souhaitons nous arrêter car elle envisage, avec les schémas de pensée propres à l'époque, les problématiques de la subjectivation des discours et de l'objectivation de la pensée, problématiques centrales dans ce que nous nommons aujourd'hui la « réflexivité ».

C'est l'étude du texte d'Athanase (la Vita Antonii), l'un des plus anciens que la littérature chrétienne nous ait laissés sur l'écriture spirituelle, qui oriente l'analyse menée, dans l'un de ses derniers articles, par Michel Foucault, vers le rôle de l'écriture dans la culture philosophique de soi juste avant le christianisme. Pour Athanase en effet, (retraçant et commentant la vie de l'anachorète égyptien Saint Antoine), la notation écrite des actions et des pensées représente un élément indispensable de la vie ascétique parce que l'écriture remplace les regards des compagnons d'ascèse. Ainsi, « écrivant nos pensées comme si nous devions nous les communiquer mutuellement, nous nous garderons mieux des pensées impures par honte de les avoir connues $»^{4}$. L'écriture de soi apparaît comme complémentaire de l'anachorèse en ce qu'elle pallie les dangers de la solitude et donne ce qu'on a fait ou pensé à un regard possible. Ainsi, explique Foucault, « ce que les autres sont à l'ascète dans une communauté, le carnet de notes le sera au solitaire » (Foucault, 1983, 4). La notation monastique des expériences spirituelles doit permettre de débusquer de l'intérieur de l'âme les mouvements les plus cachés de manière à pouvoir s'en affranchir.

D'autres fonctions marquent cette écriture. Son lien étroit avec le compagnonnage, son point d'application aux mouvements de la pensée, son rôle d'épreuve de vérité rappellent cette culture philosophique de soi qui, deux cent ans avant le christianisme, démontrait les nécessités de l'askèsis, (ou entrainement de soi par soi), dans lequel l'écriture (pour soi et pour

\footnotetext{
${ }^{4}$ Saint Athanase, Vie et conduite de notre père Saint Antoine, trad.Lavaud, § 55, cité par Foucault, p. 3.
} 
autrui) jouait un rôle considérable. Déjà Epictète, qui n'a pourtant donné qu'un enseignement oral, associait l'écriture à la méditation en ce qu'elle permet à la pensée de se préparer à affronter le réel. On pouvait avoir recours à cet exercice de la pensée sur elle-même de deux manières différentes. Soit de manière linéaire : de la méditation, à l'activité d'écriture, au gumnazeïn ${ }^{5}$, soit de façon circulaire (méditation - relecture - prise de notes, et inversement).

Dans les deux cas, l'écriture constituait « une étape essentielle dans le processus auquel tend toute l'askésis : à savoir l'élaboration des discours reçus et reconnus comme vrais en principes rationnels d'action » (ibid., 6). S'il veut en faire un élément de l'entraînement de soi, le scripteur peut se saisir de l'écriture comme d'un opérateur de la transformation de la vérité en éthos. On parlera alors avec Plutarque d'une fonction ethopoietique de l'écriture. Cette notion met le doigt sur le rôle du scripteur dans son appropriation des savoirs. Quelle médiation, quelle subjectivation doit-il opérer ? Comment? Pour qui ? A ces questions, les pratiques en cours aux Ier et $\mathrm{II}^{\circ}$ siècles apportent deux réponses : les hypomnemata et la correspondance.

\section{L'usage des hypomnemata : intégration et dépassement des savoirs}

L'usage des hypomnemata comme carnet de notes, livre de vie ou guide de conduite, s'est largement répandu auprès d'un public cultivé qui y notait des choses lues, vues ou entendues : citations, fragments, remarques ou raisonnements tenus par soi ou par d'autres. Ils constituaient une mémoire matérielle des choses qui s'offraient ainsi comme un trésor destiné à la relecture et à la méditation ultérieure. En effet, ces notes n'avaient pas pour vocation de servir d'aide-mémoire au scripteur, qui ne devait pas y avoir recours pour pallier une mémoire défaillante. Il devait effectuer un certain nombre d'exercices (lire, relire, méditer, s'entretenir avec soi-même et avec d'autres) afin qu'elles constituent une étape et un relais importants dans la subjectivation des discours. Trois objectifs règlent la pratique des hypomnemata.

Tout d'abord il s'agit non de dire le non-dit, mais de capter au contraire le déjà-dit, c'est-à- dire de « faire de la recollection du logos fragmentaire et transmis par l'enseignement, l'écoute ou la lecture, un moyen pour l'établissement d'un rapport de soi à soi aussi adéquat et achevé que possible » (Foucault, op.cit., 9). L'éthique sous-jacente à cette démarche provient d'une tension entre la pratique des citations, légitimées par la tradition et l'autorité, et un souci de soi dont les objectifs étaient d'amener la personne à se retirer en elle, à s'atteindre, à profiter et jouir d'elle-même. Il s'agissait donc avec les hypomnemata de limiter les lectures en associant systématiquement la lecture et l'écriture et de «tempérer l'une par le moyen de l'autre $»^{6}$. Cette limitation permettait de lutter contre l'éparpillement qui risque de gagner l'homme cultivé face à l'ampleur des savoirs, le rend curieux de nouveautés et l'empêche de se donner un point fixe dans la possession d'une vérité acquise. Le recueil de notes offrait un support à une réflexion plus poussée.

Le deuxième objectif de l'écriture des hypomnemata était d'amener le scripteur à une pratique réglée et volontaire du disparate. On encourageait ainsi le lecteur-scripteur à établir des passerelles entre des lectures hétérogènes plutôt que de s'enfermer dans une prétendue unité doctrinale. A ce souci de faire effectuer des choix parmi l'ensemble des possibilités offertes s'ajoutait le souci « de combiner l'autorité traditionnelle de la chose déjà dite, avec (d'une part) la singularité de la vérité qui s'y affirme et (d'autre part) la particularité des circonstances qui en déterminent l'usage » (Foucault, op.cit. ,11). «De tout ce que tu auras parcouru, extrais une pensée à bien digérer ce jour-là », conseille Sénèque à Lucilius, avant d'ajouter :

\footnotetext{
5 C'est-à-dire à l'entrainement (ou épreuve ) en situation réelle.

${ }^{6}$ Comme l'indique Sénèque, dans les Lettres à Lucilius.
} 
«C'est aussi ce que je fais. Entre plusieurs textes que je viens de lire, je jette sur l'un d'eux mon dévolu.Voici mon butin d'aujourd'hui ; c'est chez Epicure que je l'ai trouvé, car j'aime aussi à passer dans le camp d'autrui. Comme transfuge ? non pas ; comme éclaireur ${ }^{7}$.

Ceci conduit au troisième objectif de cette pratique : elle réaffirme le pouvoir unificateur de l'écriture ou, plus exactement, souligne la cohérence qu'elle permet d'opérer par l'assimilation, la digestion ou encore la subjectivation. C'est encore Sénèque qui recommande à Lucilius : «Ne souffrons pas que rien de ce qui entre en nous demeure intact, de peur qu'il ne soit jamais assimilé. Digérons la matière : autrement elle passera dans notre mémoire, non dans notre intelligence $»^{8}$.

L'acte d'écrire devient donc un acte d'appropriation des vérités contenues dans les choses lues, vues ou entendues. Par cet acte, le scripteur transforme en principe d'action rationnelle ce qui n'était, au moment de la lecture, qu'un élément de savoir. Le déjà dit hétérogène et fragmentaire, acquiert cohérence et signification par le geste scripturaire et la subjectivation qu'il a pour fonction de mettre en oeuvre. Inversement, le scripteur constitue sa propre identité à travers cette recollection de choses dites. Par le choix de ses lectures et la pratique de l'écriture assimilatrice, l'intellectuel ne fait pas seulement valoir et reconnaitre son statut de lettré ; il constitue sa propre âme (Foucault, op.cit., 13). Ainsi, le praticien des hypomnemata vise plus la qualité d'un mode d'être, (dans son écoute des autres, ses actions, ses raisonnements, ses écrits, etc.) que la possession des savoirs. Il sera jugé par ses pairs non pas en fonction des références mémorisées et citées mais en fonction de son plus ou moins grand sens de la dialectique pour se constituer comme sujet d'actions rationnelles par l'appropriation, l'unification et la subjectivation d'un "déjà dit » fragmentaire et choisi. Cette dynamique qu'instaure le praticien des hypomnemata entre intégration et dépassement des savoirs établit la possibilité d'une évolution de sa pensée et de son action dans le cadre de l'existence quotidienne.

Mais cette évolution du sujet ne saurait exister pleinement sans l'exhortation ou la stimulation exercées par autrui. L'entrainement de soi que suppose l'askésis ouvre à la figure de l'autre un espace dans lequel il va devoir occuper un certain nombre de fonctions. Une brève analyse de la correspondance telle qu'elle apparait dans ce contexte peut nous permettre d'approfondir les points qui viennent d'être abordés (le rapport qu'établit le sujet, par le biais de la lecture et de l'écriture, aux savoirs constitués en dehors de lui) et d'ouvrir la réflexion sur la question de la réciprocité.

\subsection{Correspondance, altérité et recherche de réciprocité}

Ce qui caractérise ici la correspondance c'est tout d'abord qu'elle constitue une nouvelle occasion pour le destinateur de s'exercer à l'écriture et à la (re)lecture, et qu'elle agit, sur celui qui la reçoit et la lit. Cette double incidence de la missive est assumée et exploitée notamment dans la littérature épicurienne pour ouvrir sur la notion de réciprocité, notion qui comporte deux aspects. Tout d'abord la praemeditatio. Ainsi, les Lettres de Sénèque à Lucilius montrent comment un homme déjà âgé et retiré exerce une direction sur un autre qui exerce d'importantes responsabilités publiques. Mais cette sollicitude de Sénèque n'est jamais conçue comme un acte unilatéral qui consisterait seulement à prendre des nouvelles de la vie et des progrès de Lucilius, lui prodiguer des conseils et lui rappeler quelques principes de conduite. Là n'est pas la fonction d'une écriture qui, selon Sénèque, repose sur deux principes. Premièrement, il faut s'entrainer toute sa vie et deuxièmement, on a toujours besoin de l'aide

\footnotetext{
${ }^{7}$ Lettres de Sénèque à Lucilius, cité par M. Foucault, p.11

${ }^{8}$ Cette lettre 84 de Sénèque à Lucilius constitue, selon Foucault «comme un petit traité des rapports entre lecture et écriture »(ibid.).
} 
d'autrui dans l'élaboration de l'âme sur elle-même. Dans la mesure où, «qui enseigne s'instruit, (...) les avis que l'on donne aux autres dans l'urgence de leur situation sont une façon de se préparer soi-même à une semblable éventualité $»^{9}$. Le premier aspect de ce principe de réciprocité inhérent à la lettre réside donc dans la praemeditatio qu'elle permet d'effectuer au scripteur. Celui-ci, tout occupé à réactiver les arguments qui aideront son destinataire à faire face à une situation douloureuse ou simplement épineuse, renforce sa préparation pour le cas où un événement de ce genre lui arriverait. "L'écriture qui aide le destinataire, arme le scripteur - et éventuellement les tiers qui la lisent » (Foucault, op.cit., 14). Mais la réciprocité consiste également à instaurer une relation, à l'intérieur de laquelle chacun des partenaires pourra tour à tour, prodiguer conseils et exhortations à l'autre. Le rôle de directeur de conscience que joue Sénèque vis-à-vis de Lucilius évolue ainsi au fil de la correspondance pour arriver au point où ce dernier peut à son tour exhorter et stimuler le vieil homme en lui prodiguant ses conseils en retour. Ce qui amènera Sénèque à s'exclamer : «L'habileté du lutteur s'entretient par l'exercice de la lutte; un accompagnateur stimule le jeu du musicien. Le sage a besoin pareillement de tenir ses vertus en haleine ; ainsi, stimulant luimême, il reçoit encore d'un autre sage du stimulant $»^{10}$. On retiendra ici que la réponse est complètement investie par cette préoccupation pour la réciprocité. En effet, dans la rédaction de sa lettre, le destinateur, écrivant pour l'autre, (voire au service d'autrui), se prépare, s'entraine et écrit aussi au service de lui-même. Le correspondant, arrivé à une certaine maturité philosophique, entre à son tour dans la dynamique d'une écriture totalement orientée vers le souci d'enrichir la relation à l'autre dans le cadre du "service d'âme » nécessaire à l'élaboration d'une « technique de vie » (Foucault, ibid., 23) vers laquelle tend l'askésis. L'un des effets de cet enjeu de réciprocité est repérable dans le style épistolaire qui, selon Démétrius, ne peut être qu'un style « simple, libre dans la composition, dépouillé dans le choix des mots, puisque chacun doit y révéler son âme $»^{11}$.

\section{La lettre : face-à-face à distance, dimension morale et volonté didactique}

Le second aspect notable dans cette tradition épistolaire est le face à face à distance qu'elle aménage entre les épistoliers. La notion de face à face comporte deux composantes : une composante que l'on pourrait qualifier d'affective et une seconde composante qui renvoie à l'entrainement de soi par - et sous - le regard de l'autre. C'est ainsi que Sénèque, dans sa Lettre 40, confie à Lucilius :

"Chaque fois que ta lettre m'arrive, nous voilà tout de suite ensemble. Si nous sommes contents d'avoir les portraits de nos amis absents... comme une lettre nous réjouit davantage, puisqu'elle apporte des marques vivantes de l'absent, l'empreinte authentique de sa personne. La trace d'une main amie, imprimée sur les pages, assure ce qu'il y a de plus doux dans la présence : retrouver $\gg$.

Ce plaisir affectueux des retrouvailles nous met sur la voie d'une réciprocité qui ne se satisfait pas d'un échange de surface, fut-il un échange de conseils. L'exigence qui se fait jour ici renvoie à la conviction morale et philosophique selon laquelle « nous devons régler notre vie comme si tout le monde la regardait " ${ }^{12}$. C'est donc par la missive que l'on "s'ouvre au regard des autres et qu'on loge le correspondant à la place du dieu intérieur ». Le travail d'introspection ainsi effectué par le scripteur est moins un déchiffrement de soi par soi qu' une ouverture donnée à l'autre sur soi-même.

\footnotetext{
${ }^{9}$ Ibid., ps. 14, 15, Lettre à Lucilius $n^{\circ} 7$.

10 Ceci, note Foucault, (ibid., p.16) arrive tardivement dans la correspondance entre Sénèque et Lucilius : il faudra attendre la Lettre 109 pour lire ces lignes.

${ }^{11}$ Demetrius, De elocutione, IV, 223-235, cité par Foucault, ibid., p. 17

12 Dixit Sénèque, Lettre 40, cité par Foucault, ibid., p. 17
} 
Parvenu à ce stade de son étude, Foucault pointe un phénomène chargé de sens pour qui tente de comprendre la place et les formes de la réflexivité dans cette culture de soi. Les premiers développements historiques du récit de soi ne sont pas à chercher du côté des " carnets personnels ", (les hypomnemata), dont le rôle, rappelons-le, est de permettre la constitution de soi à partir du recueil du discours des autres. On peut en revanche les trouver du côté de la correspondance avec autrui et de l'échange du service d'âme.

Cet échange ne va pas sans une modification qualitative du contenu des lettres qui provient d'une modification qu'instaure le scripteur dans son rapport avec lui-même. Ainsi, alors que Cicéron se racontait d'abord en tant que sujet d'action, (rappelant par exemple à son frère Quintus des maximes politiques), Sénèque ou Marc-Aurèle feront le récit de leur rapport à eux-mêmes et privilégierons deux thématiques dans cette écriture du rapport à soi :

-les interférences de l'âme et du corps (les impressions plutôt que les actions),

-les activités du loisir (plutôt que les événements extérieurs) ou encore : le corps et les jours (Foucault, ibid., 18).

Ce deuxième objet de la méditation épistolaire oblige le scripteur à s'examiner et, pratique des plus salutaires, selon Sénèque, à effectuer la revue de sa journée. La lettre devient donc aussi, une manière de se présenter à son correspondant dans le déroulement de la vie quotidienne. « Raconter sa journée - non point à cause de l'importance des événements qui auraient pu la marquer, mais justement alors qu'elle n'a rien d'autre que d'être semblable à toutes les autres, attestant ainsi non l'importance d'une activité, mais la qualité d'un mode d'être - fait partie de la pratique épistolaire » (Foucault, ibid., 20). Cette revue de la journée et l'examen de conscience qu'elle vise (la qualité d'un mode d'être) et que favorise la lettre étaient des pratiques prônées par différents courants philosophiques (pythagoricien, épicurien, stoïcien). Son objectif était d'amener l'individu à réactiver les règles de comportement qu'il convenait d'avoir toujours présentes à l'esprit. Cependant, rien n'indique que cette pratique ait pris la forme d'un texte écrit.

Il semble que ce soit dans la relation épistolaire - et par conséquent pour se mettre soi-même sous les yeux de l'autre - que l'examen de conscience a été formulé comme un récit de soimême : " récit de la banalité quotidienne, des actions correctes ou non, du régime observé, des exercices physiques ou mentaux auxquels on s'est livré » (Foucault, ibid., 21).

Il y a donc conjonction entre pratique épistolaire et examen de soi, et c'est sans doute ce qui a conduit Montaigne à ne pas opter pour cette pratique. En effet, dans le cas du récit épistolaire de soi-même, « il s'agit de faire venir à coïncidence le regard de l'autre et celui qu'on porte sur soi quand on mesure ses actions quotidiennes aux règles d'une technique de vie » (Foucault, ibid., 23). Or, même s'il lit plus le Romain pour sa psychologie que pour son éthique normative, Montaigne connaît la sévérité d'une philosophie dont l'aspect le plus dur est résumé dans cette formule adressée dans la Lettre 54 à Lucilius : "nous sommes allumés comme la chandelle et soufflés comme elle, et dans ce bref intervalle nous sommes destinés à souffrir $»^{13}$.

La correspondance chez Sénèque est inséparable d'une volonté didactique. Elle lui permet d'adapter constamment son éthique à la mesure de son destinataire, un individu qui se transforme, et lui permet de prendre en considération les conditions de vie de ce dernier. Destinateur, Sénèque cherche à gagner son destinataire, et s'exerce avec lui sur les petits riens de tous les jours, par l'exemple desquels il lui montre que la liberté doit se garder des choses les plus insignifiantes. Et lorsqu'il se présente lui-même dans les situations concrètes de sa vie quotidienne, il montre comment grandit et procède la sagesse. La lettre lui permet de transmettre sa théorie morale sans faire violence à l'homme en l'arrachant à la vie publique, lui

\footnotetext{
13 Sénèque, cité par H. Friedrich, op.cit., p. 74
} 
proposant ainsi « une culture qui offre à quiconque, chevalier ou esclave, (...) la chance de la dignité morale » (Friedrich, op.cit ., 76).

\section{Intégrer et dépasser l'héritage didactique}

La question pour nous, à ce stade, est d'approfondir les raisons qui firent que Montaigne, alors qu'une abondante littérature de forme ouverte existait et lui offrait au moins ces deux supports d'expression déjà codifiés qu'étaient la lettre et le dialogue, essaya quelque chose d'autre. Qu'est-ce qui, dans la forme codifiée de la lettre telle qu'elle existait au $\mathrm{XVI}^{\circ}$ siècle, risquait d'entraver son projet d'écriture et, inversement qu'est-ce que ce projet, abouti dans la forme des Essais allait apporter aux écritures de soi ?

\subsection{De l'épître didactique à la lettre chez Pétrarque}

Nous l'avons vu, l'épître didactique de celui que Friedrich a nommé « l'essayiste » Sénèque (op.cit ., 369), puise dans la souplesse de la lettre cicéronienne qui réside d'abord, suivant la coutume générale de l'antiquité, dans son adaptation au destinataire et dans les variations de style et de ton qui en résultent. Elle tient également à la communication de la privata cotidianaque vita ${ }^{14}$ dans laquelle l'auteur trouve le loisir de rentrer en lui-même.

La lettre, reprise et modifiée par les Romains à partir de l'épître didactique grecque, est une production instable dans laquelle la réflexion individuelle, peut se déployer plus librement que dans les autres genres. Son maître romain, Cicéron, peut agiter (notamment dans ses Lettres à Atticus), «toutes les questions imaginables, politiques, philosophiques et morales, scientifiques, dans une suite sans ordre à laquelle seule l'unité de l'écrivain confère une cohérence » (Friedrich, op.cit ., 368). La tradition épistolographique ainsi fondée, outre le fait qu'elle constitue l'une des sources principales de la littérature autobiographique, se caractérise donc aussi par les tentatives qui y sont faites de penser les problèmes que rencontre l'homme dans le monde (Cicéron) et avec sa conscience (Sénèque). C'est ce qui fait dire à H. Friedrich qu'il y a bien dans la lettre quelque chose de l'essai. Cette tradition ne s'interrompra plus jusqu'au haut Moyen-Age, période pendant laquelle elle retombe dans le style du traité. Lorsque Pétrarque reprend cette tradition en redonnant une forme personnelle à la lettre, il s'inspire directement de celles de Cicéron qu'il a découvertes. Il utilise « les éléments du genre créé par Cicéron comme autant de formes d'expression assimilables d'une manière d'écrire qui fait librement alterner le style artistique (voire artificiel) et le style familier » (Friedrich, op.cit., 370). Surtout, les lettres de Cicéron explorent un vaste domaine de faits, mais en les rapportant toujours à la sensibilité et au jugement de l'auteur. Personne, revendique Cicéron, «ne doit m'interdire d'aller où il me plaît, de fuir ce qui m'inquiète, de tenter ce qui ne fut jamais essayé, de suivre des chemins riants et courts, de me hâter ou de muser, de m'écarter de ma route ou de prendre à rebours » (ibid.). La composition de ses lettres varie selon son humeur, son goût du changement, les destinataires (c'est là, nous l'avons dit, une loi antique du genre), selon la variété des sujets qu'il se plait à traiter. Pétrarque continuera à développer cette tradition de la lettre, prenant la suite de ses illustres prédécesseurs : Cicéron et Sénèque.

Pour illustrer ce point, nous nous arrêterons sur un texte important dans la vie et l'évolution de Pétrarque puisqu'il relate nous nous le point de passage entre deux modes de vie et deux conceptions de l'existence et prépare la conversion future et les deux années de retraite ascétique que le poète passera, en 1337-1338 auprès de son frère, qui avait choisi la condition

\footnotetext{
${ }^{14}$ La vie privée dans sa quotidienneté, Cicéron, Ad Quintum Fratrem, I, I, 36, cité par H. Friedrich, ibid.
} 
de Chartreux. Ce texte, publié sous le titre L'Ascension du Mont Ventoux, est une lettre adressée au théologien Dionigi Roberti da Borgo San Sepolcro, rencontré trois ans auparavant.

En 1336, alors qu'il goûte depuis dix ans la vie mondaine et raffinée de la cour papale à Avignon, Pétrarque, à 32 ans, tente l'ascension du mont Ventoux que depuis l'enfance il n'a " pour ainsi dire pas quitté des yeux ». Son exemplaire des Confessions de saint Augustin, "volume minuscule, somme de douceurs infinies " ${ }^{15}$, l'accompagne dans ses voyages, « comme s'il avait autant besoin d'un guide pour s'orienter dans le monde visible que d'un gardien pour ne pas s'égarer en soi » (Dubrunquez, 1990 :17-18). Pétrarque ouvre le précieux volume pour lire et prononce ces mots à l'intention de son frère Gherardo, (modèle en qui il puisait les ressources de sa vie morale), qui le guide dans cette ascension : "Et les hommes vont admirer les cimes des monts, les vagues de la mer, le vaste cours des fleuves, le circuit de l'Océan et le mouvement des astres et ils s'oublient eux-mêmes ». Troublé, Pétrarque ferme alors le livre, intimement convaincu que tout ce qu'il vient de lire a été écrit pour lui, non pour d'autres. Il se souvient « de ce qu'avait pensé de lui-même Augustin quand, ouvrant le livre de l'Apôtre, comme il le raconte, il lut ces paroles : Ne vivez pas dans les festins, dans les débauches, ni dans les voluptés impudiques, ni dans la lutte ni dans l'envie ». Puis il se remémore les paroles d'Antoine (rapportées par Athanase) quand, à la lecture de l'Evangile, il tombe sur ces mots : " Si tu veux être parfait, va, vends ce que tu possèdes et donne aux pauvres ; viens, suis-moi, et tu auras un trésor dans le ciel ».

Pétrarque explique alors que «de même qu'Antoine trouva dans ces paroles le terme de sa quête, de même qu'Augustin, une fois lu ce passage, refusa d'aller plus loin », de même lui, Pétrarque allait méditer silencieusement ces paroles. Il conclut sur « la stupidité des hommes, lesquels, négligeant leur part la plus noble, se fourvoient en mille chemins et se perdent en vains spectacles, cherchant à l'extérieur ce qui se pourrait trouver à l'intérieur » (Ibid., 43-44). Pour Pétrarque, c'est une véritable rencontre avec le précepte de saint Augustin : "Refuse la tentation de l'extériorité ; c'est dans l'espace intérieur de l'homme que la vérité fait résidence $»$.

Pétrarque met donc en relation sa propre expérience avec les textes de saint Augustin et d'Athanase. Il s'inscrit ainsi dans une intertextualité qu'il convient de ne pas réduire à la seule dimension religieuse. Le récit de soi que produit Pétrarque, recherchant « dans son patrimoine spirituel une règle de conduite » (Dubrunquez, op. cit.,13), est une lettre adressée au père augustinien Dionigi Roberti da Borgo San Sepolcro qui depuis trois ans l'initie à la lecture de Saint Augustin. Pétrarque soumet à Dionigi, « le stoïcien qui sait la place de chaque être dans le monde (...) ce vigilant exercice d'herméneutique médiévale auquel il ne cesse de spontanément recourir pour élucider le sens caché de son acte $»^{16}$ (Ibid., p. 18-19).

Dans cette lettre, la construction du sens passe par des procédures complexes, en partie déterminées par la doctrine herméneutique qui s'exprime au Moyen-Age, et épousant la tradition épistolaire cicéronienne que nous avons rappelée plus haut. La conjonction est ici frappante entre la nécessité intérieure de composer le récit de cette expérience spirituelle et l'urgence de le soumettre, sous sa forme épistolaire, au destinataire choisi. Les derniers mots de cette lettre soulignent cette triple nécessité : écrire vite, écrire une lettre, écrire à Dionigi.

\footnotetext{
15 Pétrarque, L'ascension du Mont Ventoux, traduit du latin par D. Montbelleo, Extrait de Familiarum Rerum Libri IV, I, Paris, Séquences , 1990, p. 41

16 La doctrine herméneutique qui s'impose à la conscience médiévale et à Pétrarque ne peut concevoir que le sens littéral d'un texte - fut-il profane -, ne cache quelque intention didactique ou morale et ne suppose un supplément de sens pour se trouver légitimé. C'est dans cette perspective aussi que Pétrarque s'évertue à découvrir l'allégorie sous le sens littéral de son ascension, afin qu'au sommet du Mont, un autre sens, moral celui-ci s'impose à lui.
} 
Une nécessité sous-tendue par le désir de convier la subjectivité de l'autre dans ce mouvement d'objectivation de l'espace intérieur :

« Dans ces pensées où balançait mon coeur, (...) je revins à la cabane d'où j'étais parti à l'aube (...). Ensuite, pendant que les serviteurs s'affairaient à préparer le dîner, je me suis retiré tout seul dans un coin de la maison pour t'écrire, à la hâte et en les improvisant presque, ces pages; je ne voulais pas, en les différant, changer de sentiments en même temps que de lieux, ni que s'éteigne mon désir de t'écrire. Tu vois donc, très aimé père, que je ne peux rien cacher de moi, moi qui mets tant de soin à te révéler non seulement toute ma vie, mais aussi mes pensées secrètes, l'une après l'autre; prie pour elles, je t'en supplie, pour que, depuis le temps qu'elles errent et hésitent, elles finissent par trouver un port, pour que, après avoir été inutilement ballotées, écartelées, elles puissent se retourner vers l'unique bien, vers la vraie certitude, vers ce qui demeure. Adieu » (Pétrarque, op. cit., 45-46).

Cet extrait montre comment Pétrarque, s'il réalise admirablement les possibilités d'expression de la lettre, reste attaché aux préceptes antiques de l'examen de conscience, à la doctrine herméneutique et à certains procédés rhétoriques. Nous savons par ailleurs, qu'avant la publication de sa correspondance, il fit disparaître certains traits que gardait la première version. Epistolier prisonnier de ces diverses contraintes, il laisse subsister dans son oeuvre épistolaire les derniers vestiges d'une crainte qu'avec Erasme et Budé, il continue d'éprouver : celle de parler sans gêne de soi.

\subsection{Le formalisme de la lettre}

Montaigne analyse très bien le rôle que joue l'écriture dans la cristallisation des contenus de conscience. Pour comprendre la nécessité d'écrire qui est la sienne, il faut se souvenir que l'écriture est à ses yeux l'unique moyen d'accéder à la réalité mouvante de sa personne et du réel qui l'entoure. C'est là l'essentiel. Aussi, laissant de côté la question du public et du lecteur, se penche-t-il avec une minutieuse délectation sur son ouvrage, car cet ouvrage c'est sa vie. «Et quand personne ne me lira, ai-je perdu mon temps de m'être entretenu tant d'heures oisives à pensements si utiles et agréables ? (...). Me peignant pour autrui, je me suis peint en moi de couleurs plus nettes que n'étaient les miennes premières » (Montaigne, II, 18, 426).

Montaigne sait que passant de la méditation muette à l'écrit, il envoie ce dernier dans le monde et le communique à autrui. Le pour autrui de l'écriture est un moyen supplémentaire de se ressaisir et de se trouver lui-même, un principe concret, une certitude tangible, un point d'accroche dans l'humanité que représente à ses yeux l'autre. L'affirmation de sa propre humanité passe par l'affirmation de l'existence de l'autre, y compris dans sa fonction de lecteur. C'est bien la notion de public et non de destinataire qui convient pour désigner les lecteurs envisagés par Montaigne. S'il a besoin, pour écrire, de la certitude qu'au moins son entourage familier le lira, il ne se sent pas doué pour la correspondance. Premièrement, parce qu'il n'a personne à qui parler (« et eusse pris plus volontiers cette forme à publier mes verves, si j'eusse eu à qui parler » dit-il). Ceci est pour lui une raison pertinente : l'absence de destinataire réel supprime une condition préalable à la sincérité. D'autre part parce qu'en tant qu' « ennemi juré de toute falsification » (Ibid., II, 13, 359), il s'insurge contre l'invention d'un destinataire, invention dans laquelle Pline Le Jeune et, dans une moindre mesure Cicéron, excellaient qui portaient après coup le nom d'un dédicataire imaginaire et publiaient ainsi des lettres non envoyées. «Car de négocier au vent, comme d'autres, je ne saurais que de songes, ni forger des vains noms à entretenir en chose sérieuse » (ibid.).

Enfin, sa critique de la lettre vise la forme artistique qui en a été faite et qui a transformé une forme d'expression directe en un " théâtre d'exercices de style creux, le genre de la vaine joliesse » (Friedrich, op.cit., 373). D'ailleurs, son style ne s'y adapterait pas dit-il, donnant $a$ 
contrario sa vision de ce que pourrait être une correspondance libérée de la prison dorée de ses contraintes rhétoriques :

«J'ai naturellement un style comique et privé, mais c'est une forme mienne, inepte aux négociations publiques, comme en toutes façons est mon langage (...) et ne m'entend pas en lettres cérémonieuses, qui n'ont autre substance que d'une belle enfilure de paroles courtoises. Je n'ai ni la faculté, ni le goût de ces longues offres d'affection et de service. (...) C'est bien loin de l'usage présent; car il ne fut jamais si abjecte et servile prostitution de présentations; la vie, l'âme, dévotion, adoration, serf, esclave, tous ces mots y courent si vulgairement que, quand ils veulent faire sentir une plus expresse volonté et plus respectueuse, ils n'ont plus de manière pour l'exprimer » (Montaigne, II, 13, 359).

La lettre ouvre une voie à Montaigne. Ses caractéristiques : « le libre vagabondage d'un sujet à l'autre, l'entrelacement du style objectif et du discours à la première personne, l'expression d'une humeur contemplative ou tout bonnement capricieuse (...), la contradiction avec soimême (...), la licence d'improviser » (Friedrich, op. cit., 372) permettent à l'auteur d'abolir sa dépersonnalisation. Montaigne, tout entier dans une pensée en opposition au formalisme et à la scolastique finissante, pouvait faire épanouir cette subjectivité moderne en l'arrachant à l'enveloppe de la forme épistolaire.

\section{Le dialogue et la subjectivité improvisante}

La lettre, comme le dit une formule héritée de l'antiquité, est un dialogue à demi.

La lettre, le dialogue et l'essai ont en commun, de permettre un style d'exposition dégagé de toute contrainte et fournissent une forme pour discuter de sujets qui ne supportent pas d'être traités systématiquement ${ }^{17}$. Montaigne apprécie le dialogue et pense que Platon l'a utilisé «à escient, pour loger plus decemment en diverses bouches la diversité et variété de ses propos fantasies " (Montaigne, II, 12). Il interprète et se réapproprie les caractéristiques d'un genre qui, à ses yeux, permet à la fois l'expression littéraire de la suspension du jugement, de l'impossibilité de fixer tant l'esprit que les choses et l'expression écrite « de la subjectivité improvisante, qui peut y représenter sa richesse et ses perspectives changeantes en les distribuant entre divers personnages » (Friedrich, op.cit., 374).

Il faut rappeler que la culture mondaine des cours, où s'épanouissait la formation laïque de l'esprit, était fondée sur la conversation, et que l'oeuvre de Giordano Bruno dont la parution était quasiment contemporaine des Essais, consistait en dialogues. Mais Montaigne, laissant de côté l'entretien fictif entre plusieurs personnes, met en lumière ce que la fiction du dialogue avait jusqu'alors masqué : « l'esprit s'entretenant avec lui-même ». Et il y parvient, même si, comme Thibaudet, on peut supposer que le dialogue Montaigne-La Boétie eût donné « une grande oeuvre, fort différente du dialogue Montaigne-Montaigne que sont les Essais » (Thibaudet, op.cit., 11). Il y avait là bien sûr la possibilité d'un échange dialogué à l'intérieur d'une correspondance. Cette possibilité est réaliste si l'on est attentif aux regrets qu'exprime Montaigne de n'avoir personne à qui parler et si l'on se souvient de la façon dont il décrit cette amitié "si entière et si parfaite" (Montaigne, I, 28, 264) entre les deux hommes de lettre, publiant dans son chapitre sur l'amitié les Vingt et neuf Sonnets d'Etienne de La Boétie. Mais cet échange aurait aussi pu résulter d'une confrontation par œuvre interposée entre celle de Montaigne et celle que La Boétie aurait pu continuer à écrire s'il avait survécu (il a une trentaine d'années lorsqu'il meurt en 1563, laissant à son ami ses livres et ses manuscrits).

Le contexte historique et littéraire offrait donc à Montaigne des formes existantes. Les productions du Moyen Age et de l'humanisme (en latin et en langue vulgaire) épousaient pour

${ }^{17} \mathrm{H}$. Friedrich rappelle à propos de l'expression "dialogue à demi" que Le Tasse, retournant la formule dans son Discorso sull'arte del Dialogo, dit ceci : «Bisogna scrivere col medesimo stile il dialogo e l'epistola, perchè il dialogo è quasi una sua parte. » ( «Dialoghi », dans : Prose, éd. C. Guasti, II, 1875, p. 247). 
beaucoup les formes du dialogue et de la lettre, parentes par leur origine comme par leur développement. Pendant que la lettre préparait des moyens d'expression à la subjectivité de l'auteur et à la diffusion des matières les plus variées, le dialogue mettait à la disposition de l'esprit sceptique « une technique d'exposition qui répondait à la multiplicité équivoque des matières par ses changements de perspective » (Friedrich, op.cit., 375) et soulignait le fait que le moi n'est jamais une donnée fixe mais s'exprime dans des formes éclatées et discontinues ${ }^{18}$. Montaigne ne s'en satisfait pas, tire parti de l'une et de l'autre et crée une autre forme.

\section{Un paradigme réflexif implique la conjonction entre expérimentation formelle et expérience de soi.}

\subsection{Au fondement humaniste de la réflexivité, l'invention d'un dispositif : les Essais de Montaigne}

Les écritures réflexives qui se sont développées dans le monde occidental recouvrent un domaine d'expressions variées, font l'objet d'une forte codification culturelle et véhiculent un héritage philosophique, éthique et religieux. Un survol de ce domaine a permis d'identifier les éléments constitutifs d'une pensée humaniste qui culminera aux XVIII ${ }^{\circ}$ et $\mathrm{XIX}^{\circ}$ siècles, avec l'affirmation de la notion d'individualité. L'éducation- et la recherche- de soi se sont notamment exprimées dans des formes littéraires regroupées sous le terme d'écritures de soi (Foucault, 1983). Elles oeuvrent à la connaissance et à la communication d'un espace durablement désigné comme espace intérieur. Plutôt que de décrire l'homme à travers les autres, elles découvrent l'humain constitutif du je d'un scripteur singulier, le pari étant que sous cette singularité réside quelque chose de l'humaine condition. Ce projet trouve un premier aboutissement avec les Essais de Montaigne qui marquent un double tournant : par rapport à la tradition philosophique des auteurs de l'antiquité gréco-latine et vis-à-vis de la pensée et de la méthodologie proposée par Saint Augustin. Avec les Essais, Montaigne ouvre la voie à une anthropologie réflexive dans laquelle la notion de point de vue sur soi, sur le monde et sur autrui revendique sa relativité à son sujet et à ses objets :

«Les Essais semblent être un dispositif discursif par quoi la pensée ne cesse de s'auto-évaluer comme processus susceptible non pas tant de «rendre raison de», que de faire droit à du « réel », c'est-à-dire essentiellement à une expérience existentielle irréductible à son seul fait, et toujours élevée à la fluidité des discours qui la scandent» (Mathias, 2006, ps. 15-16)

Notre analyse de cette œuvre a pour objectif de repérer ce qui, dans ce projet d'écriture, a permis l'émergence d'une conception du sujet et de la subjectivité dont hérite notre conception actuelle de la réflexivité. Ceci dans un contexte où, à la difficulté pour dire et penser l'homme hors des catégories dominantes, s'ajoutait une lourde codification littéraire qui rendit nécessaire la construction d'une nouvelle forme : ce fut celle de l'essai. Sa dimension novatrice apparait dans le choix qui y était fait de privilégier l'expression singulière, la subjectivité de la perception et l'objectivation relative de la pensée plutôt que l'affirmation de vérités, de règles et de lois opposées à la doxa. Elle apparaît surtout dans la tentative qui y est faite de penser la dialectique identité/altérité, au sein d'une civilisation construite sur des préceptes qui interdisaient cette dialectique.

\footnotetext{
18 C'est notamment cette valeur qu'il accorde au dialogue qui conduira Diderot à y voir la métaphore de la constitution d'un savoir ou d'une identité, et à en écrire lui-même.
} 


\subsection{Premier changement de paradigme : une anthropologie réflexive émancipée vis-à-vis de l'ordre moral}

\section{L'analyse de soi, en rupture avec la culture profane antique et la tradition religieuse.}

Montaigne a renoncé à la question de savoir ce qu'est ou ce que devrait être l'homme et l'a remplacée par une interrogation précise : que sont les hommes ? et un questionnement singulier : que suis-je ?: "Les autres forment l'homme ; je le récite et en représente un particulier bien mal formé » (Montaigne, III, 2, 20). Si l'observation du cas particulier est dotée d'une immense valeur, c'est parce qu'elle seule donne accès à l'homme réel, au labyrinthe humain. En se prenant comme objet de son étude, Montaigne produit donc l'ouvrage le plus personnel de son époque malgré l'existence de déjà nombreuses autobiographies, confessions, mémoires et lettres en tout genres. Le résultat n'est pas moral mais anthropologique. En effet, le premier objectif de cette analyse de soi est de découvrir le caractère mêlé voire contradictoire de l'être humain et non d'ériger une forme exemplaire : « car (les actions humaines) se contredisent communément de si étrange façon qu'il semble impossible qu'elles soient parties de même boutique (...). Et les bons auteurs (...) choisissent un air universel et, suivant cette image, vont rangeant et interprétant toutes les actions d'un personnage, et, s'ils ne les peuvent assez tordre, les vont renvoyant à la dissimulation » (Montaigne, II, 1, 15-16). Contrairement à ces bons auteurs qui définissent l'universalité et l'identité de l'homme en référence à un schéma moral et normatif, Montaigne se passionne pour l'observation et la description des faits humains dans leur diversité. C'est en effet dans la diversité de ses actions, de ses coutumes et de ses opinions que chacun est amené à affirmer ce qu'il a d'unique et d'irremplaçable. Deux éléments caractérisent cette posture d'écoute de la société des hommes. Tout d'abord, elle se méfie des jugements parce que « tous jugemens en gros sont lâches et imparfaits » (Montaigne, III, 1, 158) et Montaigne se " bande volontiers contre ces vaines circonstances qui pipent nostre jugement par les sens » (ibid., 146). Il s'agit donc d'être avant tout réceptif et disponible pour observer non seulement la diversité mais aussi le changement car «Le monde n'est qu'une branloire perenne » et « Je ne puis asseurer mon object. Il va trouble et chancelant, d'une yvresse naturelle » (Montaigne, III, 2, 20).

La deuxième caractéristique de cette posture d'observation est une conséquence directe du caractère changeant et diversifié du monde. L'observateur doit adopter des points de vue variés, à la fois parce que le monde qui l'entoure change et parce que lui-même ne cesse de se modifier avec le monde et selon ses propres humeurs. Cette observation n'est donc pas passive. Plus le regard se fait précis plus le travail à effectuer est actif: "L'homme s'approchant au plus près doit particulariser davantage encore l'image de l'homme déjà abondamment différenciée par l'observation objective, c'est à dire dissoudre en contradictions toute unité spécieuse» (Friedrich , 1949/1968, 222). La proposition que fait Montaigne à ses lecteurs, (et qui se réfère à la méthode sceptique), est explicitée de la façon suivante : « Si je parle diversement de moi, c'est que je me regarde diversement. Toutes les contrariétés s'y trouvent $(. .$.$) ; et quiconque s'étudie bien attentivement trouve en soi, voire et en son$ jugement même, cette volubilité et discordance. Je n'ai rien à dire de moi, entièrement, simplement et solidement, sans confusion et sans mélange, ni en un mot. Distingo est le plus universel membre de ma logique » (Montaigne, II, 1, 21).

La portée de ce propos est immense, car seule l'acquisition de cette connaissance de soi peut conduire à une résistance méthodique et volontaire aux oblitérations entrainées par la formation morale (et) le bon ton de la société. Cette pratique de quête de soi a pour but d'amener l'individu à une plus grande autonomie vis-à-vis des règles qui régissent l'ordre social, éducatif et moral : « il n'est personne, s'il s'escoute, qui ne découvre en soi une forme sienne, une forme maistresse, qui luicte contre (l'art et) l'institution, et contre la tempête des 
passions qui lui sont contraires » (Montaigne, III, 2, 26) ${ }^{19}$. La discipline de l'introspection a ici comme finalité de lutter contre diverses formes d'emprise et d'asujettissement et, n'a en cela, rien de commun avec la quête chrétienne du salut et la soumission au divin. Dans la perspective tracée par Saint Augustin, (qui caractérisera tout le courant mystique de la théologie médiévale et ultérieure), la connaissance de soi est la voie de la connaissance de Dieu. Elle est prioritaire et passe avant toutes les autres. Première expression de l'introversion occidentale qui ne cesse de s'imposer à côté de la connaissance objective du monde, elle rappelait l'homme à la méditation de soi-même. Si l'analyse de soi, spiritualisée par le christianisme, était déjà redevenue une psychologie profane avant Montaigne, c'est pourtant chez lui qu'elle a pris la forme d'une considération de l'existence personnelle contingente, sans référence à la morale. Ce point mérite que l'on s'y attarde car il détermine la réflexion que l'on peut mener encore aujourd'hui, sur l'écriture de soi en tant que réflexivité et recherche de sens, libérée de toute problématique de croyance religieuse.

Les mouvements du moi ne dessinent pas, aux yeux de Montaigne, les phases préparatoires d'une évolution de sa personne, ils ne constituent pas un agencement mystérieusement viable. A l'inverse, Montaigne se plaît à montrer que son moi est un concours de données surprenantes, ne formant jamais un tout exprimable. Son imagination créatrice s'ingénie à suivre et à représenter « les fluctuations et les antinomies de son propre chaos » (Friedrich, op.cit., 231). Il y a donc bien dans les Essais, représentation, c'est à dire « transposition de la réalité vivante dans une autre sphère de réalité, dotée de caractéristiques propres » (Gusdorf, 1991, 14), mais cette transposition n'obéit pas à la volonté de réformer le moi selon l'exigence d'un devoir être, pour reprendre la terminologie que Gusdorf utilise pour décrire l'entreprise, selon lui réformatrice, caractérisant les écritures du moi. L'inquiétude philosophique de Montaigne le protège du manichéïsme : "L'anthropologie de Montaigne est solidaire d'une ontologie; l'être de l'homme se trouve pris dans l'universel mobilisme de la création dont il ne semble pas qu'elle se trouve en progrès vers une fin bonne ou mauvaise » (ibid, 34). Le moi de Montaigne, proposé comme le centre de la personnalité, n'apparaît jamais comme un point fixe à jamais défini mais comme le point moyen de l'incessante gravitation de l'expérience individuelle: "Ainsi se trouvent conciliés un mobilisme qui semblerait justifier un impressionisme du mouvement perpétuel et un principe d'identité » (ibid., 35). Ces deux aspects, (le mobilisme et l'identité de l'être), sont magnifiquement exprimés dans deux citations restées célèbres : "Je ne peints pas l'être. Je peints le passage : non pas un passage d'âge en autre ou, comme dict le peuple, de sept en sept ans, mais de jour en jour, de minute en minute (...). Si mon âme pouvoit prendre pied, je ne m'essaierois pas, je me résoudrois; elle est tousjours en apprentissage et en espreuve ». Et, quelques lignes plus bas, Montaigne déclare : «Je propose une vie basse et sans lustre, c'est tout un (...); chaque homme porte la forme entière de l'humaine condition » ( III, 2, 20). Nous reviendrons plus longuement sur la forme des Essais, mais on peut déjà remarquer que Montaigne s'écarte des caractéristiques d'une autobiographie typique, qui interprète la vie personnelle « comme une structure formant un tout cohérent dont le sens se dégage de phase en phase, et pourvue d'un contenu qu'il serait possible de représenter en totalité, en y incluant les circonstances historiques extérieures » (Friedrich, op. cit., 236). Le renoncement à l'ordre achevé de la forme rend d'autant plus frappant le fragment, au sein duquel brillent les énigmes du monde et de la personnalité de l'auteur. D'ailleurs, ce renoncement exprime une position épistémologique au sujet de la perception de la vérité « objective ».

\footnotetext{
19 André Gide, dans sa Préface de 1962 à l'édition Gallimard du tome I des Essais, note que Montaigne a supprimé plus tard " contre l'art», «pour ne laisser debout, à lutter contre, que « l'institution », soit, au sens où l'on prenait alors ce mot : l'éducation (...). C'est-à-dire qu'il admet que notre éducation travaille, aussi bien qu'à nous élever, à nous contrefaire » (Gide, 1962, 11).
} 


\subsection{Second changement de paradigme : la scrutation du réel et l'affirmation d'un point de vue éthique et politique}

Si l'exercice des charges publiques représente pour Montaigne la concession extrême qu'il accorde aux exigences institutionnelles, il est une activité qui étanche sa soif d'observation et de contact avec la réalité, et cela, sans s'y perdre lui-même : l'expérience du voyage ${ }^{20}$. Cet élargissement de l'horizon humain, cette connaissance de l'homme à travers l'espace et le temps du voyage relèvent de la manière dont le $\mathrm{XVI}^{\circ}$ siècle a mené son expérimentation en matière de science morale : on découvre la réalité humaine dans la perspective de son inclassable et infinie diversité. Montaigne prend personnellement part à cette enquête et tente, lors de son voyage de 1580 , de percevoir le réel aussi exactement que possible. Il apprend l'italien afin d'entrer par imitation dans l'intimité de l'étranger et pour l'essayer personnellement et recueille dans son journal de voyage des matériaux qu'il reprendra en partie dans les Essais. Le voyage constitue ainsi «la forme d'expérience appropriée à la pensée, laquelle préfère, sceptique, se perdre dans la richesse inépuisable du réel plutôt que, dogmatique, fermer les yeux sur elle » (Friedrich, op.cit., 268). C'est donc ici que s'exprime le mieux cette anthropologie descriptive qui privilégie l'examen sceptique (c'est-à-dire scrutateur), des faits et de leurs particularités variables et abandonne la classification selon des maximes et des normes morales qui présupposent la constance ou la répétition des situations et des individus. Ainsi, « l'observation de l'homme passe du schématisme des archétypes à la notation instantanée des traits individuels » (ibid., 160). En déclarant que " tout jugements en gros sont laches et imparfaicts », Montaigne affirme que toute classification est erronée parce qu'elle repose sur une échelle de valeurs préconstruite et donc inadéquate à son objet et parce qu'elle déduit le particulier (l'homme concret) de quelque généralité.

Pour bien saisir la double portée, politique et éthique, de cette affirmation, il convient de se rappeler que, quelques années plus tôt (le 20 janvier 1531 très exactement), dans la lettre qu'il destinait à Charles Quint, empereur et roi d'Espagne, un homme énonçait une idée radicalement moderne : l'idée du respect de l'autre. Cet homme, Bartolomé de Las Casas, un prêtre devenu frère dominicain, ancien colon, était défenseur des Indiens et admirateur de l'Amiral. Pour convaincre, il accumule les preuves, raconte les massacres dont il a été le témoin, dénonce les horreurs perpétrées par les conquérants et qui aboutirent à la destruction des Indes ${ }^{21}$. Il proclame jusqu'à sa mort, en 1566, l'égalité des hommes et le refus des conquêtes. Penseur et politique, il « introduit un critère de relativité qui ébranle la hiérarchie entre les peuples. Non seulement l'autre n'est pas méprisable parce que différent, mais, de plus, nous ne saurions oublier cet autre que nous fûmes nous-mêmes » (Plenel, 1992, 120124). Las Casas argumente : «Car la plupart des nations du monde, sinon toutes, furent bien plus perverties, irrationnelles et dépravées ». Poussant le raisonnement plus loin, il en arrive à questionner la légitimité des valeurs et des croyances des peuples occidentaux. Pour cela, il aborde le point central de l'argumentation de ses adversaires, à savoir les rituels cannibales des Indiens des Petites Antilles. Affirmant que chacun a ses propres valeurs, il se décentre visà-vis de son cadre de référence et adopte une démarche compréhensive : "Les nations qui offraient des sacrifices humains à leurs dieux montraient ainsi, en idolâtres fourvoyés, la haute

\footnotetext{
${ }^{20}$ Montaigne fit son plus grand voyage en 1580/1581, après la parution des deux premiers livres des Essais. Il gagna l'Allemagne du Sud, atteignit l'Italie où il voulait guérir sa gravelle aux eaux. Il tint un journal qui ne parut que 200 ans plus tard, en 1774, sous le titre Journal de voyage de Michel de Montaigne

${ }^{21}$ Sa Très brève relation de la destruction des Indes dresse le catalogue « d'horreurs vécues où des mères sont embrochées avec leurs enfants, des bébés fracassés sur des rochers, des femmes enceintes éventrées, des lévriers dressés à dévorer à vif, des caciques brûlés à feu doux sur des grils... » (cité par Plenel, 1992,120). Ses autres livres ne seront, pour la plupart, édités qu'au XIX ${ }^{\circ}$ siècle.
} 
idée qu'elles avaient de la valeur de leurs dieux ». La conclusion de Las Casas tombe : "Tout peuple, si barbare qu'il soit, peut se défendre des agressions d'un peuple plus civilisé qui prétendrait l'asujettir ou le priver de sa liberté (...). Cette guerre est plus juste que celle qu'on lui fait sous prétexte de supériorité culturelle » (Cité par Plenel, ibid.,122).

Cinquante ans après, Montaigne ayant lu les récits des voyageurs, et rencontré en 1562, à la cour de Charles IX en séjour à Rouen trois Indiens « bien misérables de s'être laissés piper au désir de la nouvelleté » écrivait « qu'il n'y a rien de barbare et de sauvage en cette nation, à ce qu'on m'en a rapporté, sinon que chacun appelle barbarie ce qui n'est pas de son usage ». Et il ajoute : " comme de vrai, il semble que nous n'avons autre mire de la vérité et de la raison que l'exemple et idée des opinions et usances du pays où nous sommes. Là est toujours la parfaite religion, la parfaite police, parfait et accompli usage de toutes choses ». Comme Las Casas, il dénonce l'aveuglement des Occidentaux vis-à-vis de leurs propres horreurs barbaresques, aveuglement d'autant plus grave que nous «surpassons (ces peuples) en toute sorte de barbarie ». Rapportées aux processus de production des Essais, ces prises de position de Montaigne expriment une donnée fondamentale de sa pensée à savoir ce «scepticisme clairvoyant » qui caractérise son " sens de la diversité humaine dans l'espace et le temps » (Friedrich, op.cit., 123). Cette faculté se traduit par deux aptitudes : la tolérance (vis-à-vis notamment de toutes les formes de religion qu'il considère comme des faits intéressants de l'histoire des civilisations), et le sens de la relativité du jugement. Ainsi, parvenu au terme de son essai sur les cannibales, il relate la conversation qu'il eut avec trois Indiens à la cour de Charles IX et rapporte leurs propos. Ceux-ci, répondant à la question : "Qu'avez-vous trouvé de plus admirable à la cour? » dirent :

«qu'ils trouvaient en premier lieu fort étrange que tant de grands hommes, portant barbe, forts et armés, qui étaient autour du Roi (il est vraisemblable qu'ils parlaient des Suisses de sa garde), se soumissent à obéir à un enfant, et qu'on ne choisisse plutôt quelqu'un d'entre eux pour commander ; secondement, (ils ont une façon de leur langage telle, qu'ils nomment les hommes moitié les une des autres) qu'ils avaient aperçu qu'il y avait parmi nous des hommes pleins et gorgés de toutes sortes de commodités, et que leurs moitiés étaient mendiants à leurs portes, décharnés de faim et de pauvreté; et trouvaient étrange comme ces moitiés ici nécessiteuses pouvaient souffrir une telle injustice, qu'ils ne prissent les autres à la gorge, ou missent le feu à leurs maisons » (Montaigne, I, 31, 303-314).

L'ouverture d'un dialogue avec lui-même permet à Montaigne d'entendre, de comprendre et de transmettre par l'écriture, le dialogue qu'il a, au cours de sa vie, noué avec d'autres hommes en faisant preuve de cette qualité que l'on nomme aujourd'hui empathie. Autrement dit, l'écoute qu'il entretient vis-à-vis de lui-même est indissociable de celle qu'il cultive vis-à-vis de son temps. Il comprend son époque au point de penser en avance sur celle-ci : " cent ans quand il s'agit de la sorcellerie, deux cents quand il s'agit des lois, trois cents quand il s'agit de l'éducation ", évalue Albert Thibaudet dans sa Préface à l'édition de 1962 du tome 2 des Essais. Ce double dialogue fait entrer les idées dans le champ littéraire, « moins par leur force logique que par leur humanité » (ibid., 8). Le projet que ne cesse de rappeler Montaigne : « Ce sont ici mes fantaisies, par lesquelles je ne tâche point à donner à connaître les choses, mais moi » (Montaigne, II, 10), sous-tendu par un parti pris stratégique d'humilité et d'abaissement de soi, crée l'espace nécessaire à l'émergence d'une littérature d'idées. Comme le souligne Thibaudet, le moi vivant de ce premier homme moderne que nous connaissions par le détail, était d'abord un lieu d'idées. Rien d'étonnant alors à ce que ce moi soit également innovant : affirmer à ce point sa présence à soi et son ouverture au monde des idées, plaider pour que l'on pense la vie comme une "fricassée » d'exercitations et de " coups d'essay » ne pouvait aboutir qu'à la tentative de penser, d'écrire et de communiquer différemment ses savoirs et ses apprentissages. D'autant plus que Montaigne, tel Héraclite, se méfiait du langage. 


\subsection{Troisième paradigme : un autre rapport au langage}

Le rapport que formule Montaigne vis-à-vis du langage, disséminée dans l'ensemble de son ouvrage, soutient sa représentations héraclitéenne du monde. Le langage et son objet sont l'un et l'autre mouvants par eux-mêmes ; "ils ne peuvent se rapprocher qu'un instant fugitif, et toujours le langage comporte le danger de transformer l'originalité, la différence, en une identité apparente avec autre chose et de l'y anéantir » (Friedrich, op.cit., 169).

L'essentiel de la question philosophique des Essais serait donc celle de la «jointure » entre les mots et les choses, «qui n'est précisément «jointure » que par métaphore, parce que l'un et l'autre ordres ne sont ni en regard ni en écho, mais bien plutôt expressifs d'une même pléthore de l'être et du penser » (Mathias, 2006, 18). Si les Essais sont une problématisation du réel et questionnent sa dicibilité, il se trouve que, d'une nature à la fois ontologique et linguistique, la problématicité du réel réside dans sa pluralité. «La seule variété me paie, (écrit-il dans «De la vanité ») et la possession de la diversité ». Car la satisfaction que provoque l'expérience du multiple tient chez Montaigne « à la coalescence de la diversité de ce qui paraît être, et de la diversité de l'expérience intellectuelle et énonciative de ce qui est coalescence de l'apparaître dans son devenir, et de la pensée dans son dire » (ibid.).

Si Montaigne s'énonce «le premier par son être universel : comme, Michel de Montaigne ». la rencontre de l' universel et du singulier, c'est le tissu indéfini des concrétions textuelles dont porte témoignage le texte indéfiniment retravaillé des Essais, texte d'une entièreté perpétuellement questionnée. Il ne faut par conséquent pas interpréter de manière prosaïque cette idée que « chaque homme porte la forme entière de l'humaine condition ». On jugera que s'il ne s'agit que d'une forme, il revient à l'individuel et au singulier d'en être l'effectivation, c'est-à-dire la pluralisation. Dans cette optique, on comprendra mieux la distance que voit Montaigne entre son entreprise egologique et celle des moralistes : tandis que les autres forment l'homme, lui le « récite». Eux postulent l'universel et cherchent à l'abstraire, lui rencontre le singulier pluriel et le réalise en son entièreté textuelle. Peut-être faut-il donc en venir à imaginer «qu'en effet Montaigne n'a de réalité, pour lui-même comme pour nous, qu'en tant que cette aspiration à de l' " universel », comme parole textuelle et proliférante, critique, contradictoire, redondante, récurrente, réajustée, assertive ou interrogative, sceptique et dogmatique, protéiforme en tout cas et pour reprendre une de ses expressions : «effectuelle ». Et qui s'offre très simplement: «comme les enfants qui proposent leurs essais, instruisables, non instruisants » $(\mathrm{I}, 56,523)$.

On relèvera deux conséquences à cette conception du langage. Il faudra tout d'abord préférer l'image (les anecdotes, le récit) à l'interprétation abstraite et ne pas hésiter à exprimer de différentes manières une même idée. Ainsi, le parler découlant de la vision plastique, l'écrivain se renouvelle sans cesse en renouvelant ses points de vue. Il convient également, face à cette fluctuation du langage, de se souvenir que l'homme concret, n'est saisissable du dehors que de façon approximative car ce qu'il est réellement, il ne l'est que pour soi. Pour découvrir l'homme, Montaigne sait alors qu'il n'a de sol ferme qu'en se prenant lui-même pour objet. La vigilance qu'exerce Montaigne pour ne pas rater ou figer cet objet est le creuset dans lequel se forme sa conscience littéraire. C'est pourquoi il passe sans cesse de l'étude de soi au minutieux examen de son activité d'écriture : "Méditer sur sa propre nature, méditer sur sa pensée, méditer sur l'expression littéraire de l'une et de l'autre, ce sont les phases d'une seule et même opération. La conscience de l'écrivain fait ici partie, dans des proportions jusqu'alors inconnues, de la vision qu'il se donne de son individu » (Friedrich, 340).

Cette convergence entre l'émergence d'une subjectivité en littérature et celle d'une forme ouverte nous intéresse particulièrement. Chez Montaigne, l'écriture de soi, « consubstantielle à son auteur », pour le paraphraser (II, 426) ne peut se passer de cette incessante réflexion sur 
ce qu'elle est. L'expression de soi est ici inséparable du questionnement régulier de la forme qui permet cette expression. On peut se demander si, inversement, cette interrogation réflexive sur le "genre" n'est pas indispensable pour diriger et faire avancer le projet de l'étude de soi et, à travers elle, l'étude de l'homme.

\section{Conclusion}

La codification culturelle des écritures du moi jusqu'au $\mathrm{XVI}^{\circ}$ siècle et aux Essais met deux éléments en lumière. Premièrement, la forme ouverte ne se donne pas d'emblée comme le genre de l'expression subjective et, par conséquent, la lettre n'ouvre pas en elle-même à la réflexivité et à la relation intersubjective. Ou plutôt, l'instauration d'un dialogue avec soi et avec un destinataire passe par une modification profonde de la conception du sujet et des modes d'accès au « moi ». La relation à soi et à l'autre est marquée par un certain nombre de préceptes et de devoirs d'ordre théologiques et philosophiques. Le travail de Montaigne permet de commencer à libérer le "je" du moule didactique et théologique qui lui avait donné naissance dans la tradition littéraire occidentale. Il ouvre ainsi d'autres possibilités à cette forme de communication que sera l'essai.

On l'a vu, cette évolution ne résulte pas d'une rupture. Une série d'événements éthiques, esthétiques, politiques et culturels font que l'homme de la Renaissance peut penser autrement diverses questions (Kauffman, 2001). En premier lieu, celle de la perception et du point de vue (relatifs et déterminés culturellement ) et celle de l'existence du moi affirmée comme un postulat. Libéré du devoir de progresser dans la connaissance d'un Dieu transcendant, ce moi occupé à l'étude de ses imperfections, s'individualise dans l'immanence de son rapport à autrui et au monde. Plaçant l'existence elle-même sous le signe de l'essai, Montaigne exprime l'expérience, la tentative, l'exercitation. Dès lors une triple thématique va se déployer dans cet espace ouvert par les Essais : la réflexion sur soi (les événements de la conscience et de la vie personnelle), la réflexion sur le monde (la perception des événements et des idées) et la réflexion sur l'acte d'écrire (le sens de cet acte).

Ainsi, la recherche du sens à laquelle contribue cet écrit ne va pas sans la réflexion qu'il propose simultanément sur le sens de cette recherche. Problématique essentielle qui assure la vitalité d'un genre se renouvelant singulièrement sous la plume de chaque scripteur. La question du message est inséparable du questionnement sur les processus et les procédés qui permettent de l'élaborer et de le transmettre, parce que processus et procédés renseignent sur la construction des points de vue du scripteur chaque fois qu'il dit "je". L'écriture va ainsi contribuer à la construction identitaire, ou, pour le dire avec Ricoeur, aux processus de configuration de l'identité narrative.

Les écritures de soi ne naissent pas du seul rapport à l'intime ou au secret. Bien au contraire, les Essais continuent d'évoquer une raison à la fois singulière et citoyenne du monde, ni complète, ni transparente, ouverte à une pluralité d'interprétations possibles. Non pas la rationalité du siècle des Lumières qui croyait possible d'édifier un monde d'où tous les préjugés auraient disparu, mais une raison qui pénètre dans la totalité de l'existence, y compris la perception sensible, la vie affective, jusqu'à notre physiologie et qui donne au sujet la possibilité de penser et de donner un sens au caractère impermanent et transitoire du monde dans lequel il vit. Seule la reconnaissance d'une rationalité finie offre toute sa place à la subjectivité qui doit faire face à une existence vue comme mobile, chaotique et transitoire. C'est pourquoi il est si important de situer les rapports entre la rationalité et la subjectivité dans une même perspective philosophique.

Si les Essais questionnent les liens entre littératie et réflexivité, c'est bien parce que plus qu'un texte, ils constituent un dispositif permettant de tenter d'organiser l'éphémère : lire- 
écrire afin d'élaborer ses savoirs et ses techniques tout en reliant les fragments de son existence.

\section{Bibliographie}

BARRE De MINIAC, C., BRISSAUD C., RISPAIL M., (2004), La littéracie : conceptions théoriques et pratiques d'enseignement de la lecture-écriture, L'Harmattan, Paris.

CHISS, J.-L., (2004), La littératie : quelques enjeux d'une réception dans le contexte éducatif et culturel français, in: Barre de Miniac, Brissaud, Rispail, La littéracie : conceptions théoriques et pratiques d'enseignement de la lecture-écriture, L'Harmattan, ps. 43-53, Paris.

DUBRUNQUEZ, P., (1990), Préface, L'Ascension du Mont Ventoux, Pétrarque, traduit du latin par Denis Montebello, Séquences, Paris.

FOUCAULT, M., (1983), L'écriture de soi, L'autoportrait , Corps écrits n 5, P.U.F., Paris.

FRIEDRICH , H., (1968), Montaigne, A. Francke Verlag AG, 1949 ; A. Francke Verlag AG, 1967, pour l'édition revue et augmentée; éditions Gallimard, coll. NRF, 1968 pour la traduction française de R. Rovini., Paris.

GUSDORF , G., (1991), Lignes de vie I, Les écritures du moi, Odile Jacob, Paris.

HAMERS, J, (1997), Attitudes langagières et développement de la littératie chez des élèves scolarisés en français, Diversité linguistique et culturelle et enjeux du développement, Ed. Aupelf-Uref, Université Saint-Joseph, pp. 369-381, Beyrouth.

KAUFFMANN, J.-C, ( 2001), Ego, Pour une sociologie de l'individu, Nathan, Paris.

LAINE, A., (1997), Faire de sa vie une histoire, Desclée de Brouwer, Paris.

MOLINIE, M., (1993), Le choix de la lettre, in : Vers une approche relationnelle de la communication interculturelle, Doctorat en didactique des langues et des cultures, Paris 3 Sorbonne-Nouvelle, Paris.

MATHIAS, P. (2006), Montaigne ou l'usage du monde, bibliothèque des philosophes, VRIN, Paris.

MOLINIE, M., BISHOP, M.-F., (2006) Autobiographie et réflexivité, CRTF-Belles Lettres, Cergy-Pontoise.

MONTAIGNE, (1580), Essais I, éditions de 1965 pour l'établissement du texte, la présentation et l'annotation, 1962, pour la préface d'André Gide, Folio n 289, Paris.

MONTAIGNE, (1580), Essais II, 1, édition de 1965 pour l'établissement du texte, la présentation et l'annotation, 1962 pour la préface d'A. Thibaudet, Folio n²90, Paris.

MONTAIGNE, (1588), Essais II, éditions de 1965 pour l'établissement du texte, la présentation et l'annotation, 1962, pour la préface d'Albert Thibaudet, Folio ${ }^{\circ} 290$, Paris.

PETRARQUE, (1990), L'Ascension du Mont Ventoux, traduit du latin par Denis Montebello, Extrait de Familiarum Rerum Libri IV, I, Séquences, Paris.

PLENEL, E., (1992), Voyage avec Colomb, Le Monde-La Découverte, Paris.

ROBILLARD, de D., ( 2007), La linguistique autrement: altérité, expérienciation, réflexivité, constructivisme, multiversalité : en attendant que le Titanic ne coule pas, Carnets d'Atelier de Sociolinguistique ${ }^{\circ} 1$, ps. 1- 148.

ROUXEL, A. (2006), Genres autobiographiques et genres réflexifs, in : Molinié, Bishop, (dir.), Autobiographie et réflexivité, CRTF-Belles Lettres, Cergy-Pontoise, ps. 15-31. 